\title{
Are breast cancer screening programmes increasing rates of mastectomy? Observational study
}

\author{
Eugenio Paci, Stephen W Duffy, Daniela Giorgi, Marco Zappa, Emanuele Crocetti, Vania Vezzosi, \\ Simonetta Bianchi, Luigi Cataliotti, Marco Rosselli del Turco
}

\begin{abstract}
Unit of
Epidemiology,

Centre for the

Study and

Prevention of

Cancer CSPO, Via

di S Salvi 12, 50135

Florence, Italy

Eugenio Paci

director

Marco Zappa

senior epidemiologist

Emanuele Crocetti

senior epidemiologist

Screening Unit,

Centre for the

Study and

Prevention of

Cancer CSPO

In the recent Cochrane review of mammographic screening the authors and editors disagreed with respect to reporting the effect of screening on rates of aggressive treatment for breast cancer. ${ }^{1}$ The authors maintained that screening increased the number of mastectomies by around $20 \%$, mainly as a result of overdiagnosis. If this were the case, populations in which screening has been introduced should see a subsequent increase in the number of radical treatments for breast cancer and an increase in the incidence of breast cancer in excess of that expected purely from lead time. We report the changes in rates of radical surgery and incidence of breast cancer since the introduction of the Florence mammographic screening programme.
\end{abstract}

Marco Rosselli del

Turco

director

Dept of

Mathematics,

Statistics and

Epidemiology,

Imperial Cancer

Research Fund,

London

WC2A 3PX

Stephan W Duffy

principal scientist

Unit of

Epidemiology,

Azienda Sanitaria

Locale (ASL) 2,

55100 Lucca, Italy

Daniela Giorgi

senior epidemiologist

Pathology

Department,

University of

Florence, 50134

Florence, Italy

Vania Vezzosi

pathologist

Simonetta Bianchi

senior pathologist

Surgery

Department,

University of

Florence

Luigi Cataliotti

professor of surgery

Correspondence to:

E Paci

Epid1@cspo.it

BMJ 2002;325:418

\section{Methods and results}

We studied the period from 1990 to 1996 . During this time 59947 women aged 50-69 years were invited to a prevalence screen and to subsequent screens at two year intervals (2.4 years in practice). ${ }^{2}$ At the beginning of 1990 no women had been invited; by the end of 1994 all the women had been invited to at least one screen. Attendance rates averaged $60 \%$. We considered cases of breast cancer with pathological size $T_{2}$ or greater to be late stage. Early stage cases included tumours of less than $2 \mathrm{~cm}$ size and in situ carcinomas.

We plotted absolute rates of mastectomy and breast conserving surgery-defined as lumpectomy, wide excision, and quadrantectomy-per thousand women in the population (not per cancer case) against time for the years 1990-6. We assessed the significance of changes over time by trend tests in Poisson regression.

The figure shows the absolute rates of mastectomy and breast conserving surgery over time, with the disease incidence by stage. Rates of breast conserving surgery increased by around 0.7 per thousand $(\mathrm{P}=0.01)$ over the period 1990-6, and rates of radical surgery declined by 0.5 per thousand $(\mathrm{P}<0.001)$. These rates closely paralleled those of early and late stage breast cancer. Early disease and breast conserving surgery peaked in 1993 as the mobile screening units completed the prevalence screen; this was followed by a reduction in 1994 after the prevalence screen had finished, as one would expect. ${ }^{3}$ The rates were also strongly related to the amount of screening activity. The peak number of mammograms was 17298 in 1993, followed by only 8965 in 1994 when the prevalence screen was largely completed but the first repeat screen only just beginning. In 1990 the rate of mastectomy was 1.08 (95\% confidence interval 0.84 to 1.37 ) per thousand, whereas in 1996 it was 0.62 ( 0.44 to $0.86)$ per thousand. The rates of breast conserving surgery were 1.18 (0.92 to 1.48 ) per thousand in 1990 and 1.87 (1.54 to 2.21) per thousand in 1996.

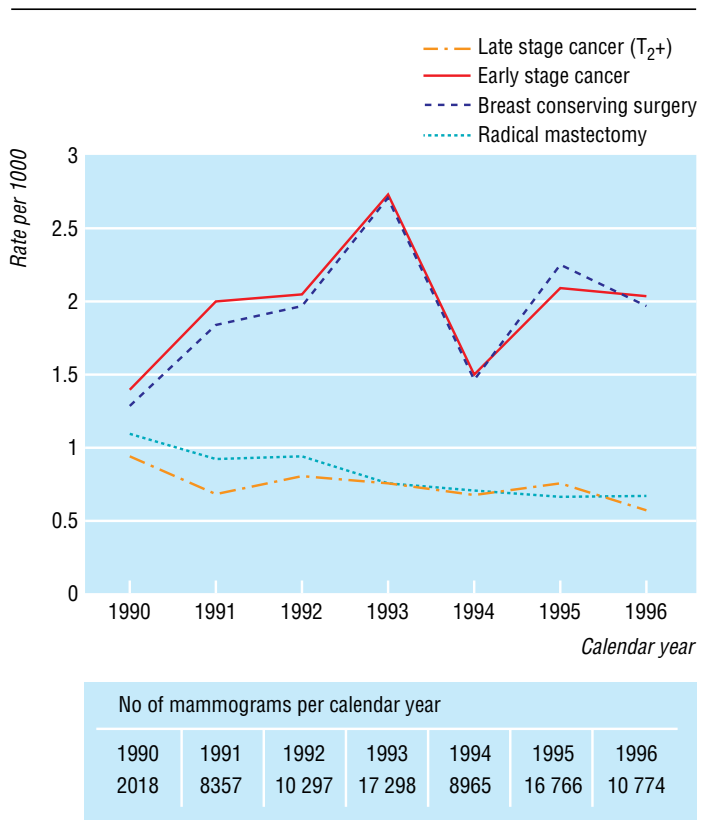

Size of breast cancers and type of operation

\section{Comment}

The rate of breast conserving surgery has increased significantly with the advent of screening, and the rate of radical surgery has declined significantly. Similar reductions in mastectomy rates have been observed elsewhere. ${ }^{4}$ This indicates that the introduction of screening brings about a reduction in mastectomy rates, not an increase. Follow up will continue to ascertain whether these findings are maintained.

Contributors: EP, DG, and MZ did the epidemiological analysis. SWD did the statistical analysis. VV, SB, LC, and MRdelT reviewed and interpreted the pathological, surgical, and radiological data. EP and SWD wrote the paper. All the authors contributed to the final version. EP is the guarantor.

Funding: EP was supported by an American Cancer Society grant. The Florence City Programme is supported as a member of the European Breast Cancer Screening Network.

Competing interests: None declared.

1 Olsen O, Gotzsche PC. Cochrane review on screening for breast cancer with mammography. Lancet 2001;358:1340-2

2 Paci E, Duffy SW, Giorgi D, Prevost TC, Rosselli del Turco M. Populationbased breast cancer screening programmes: estimates of sensitivity, overdiagnosis and early prediction of the benefit. In: Duffy SW, Hill C, Esteve J, eds. Quantitative methods for the evaluation of cancer screening. London: Arnold, 2001.

3 Day NE, Walter SD. Simplified models of screening for chronic disease: estimation procedures from mass screening programmes. Biometrics 1984;43:1-13.

4 Wingo PA, Guest JL, McGinnis L, Miller DS, Rodriguez C, Cardinez CJ, et al. Patterns of inpatient surgeries for the top four cancers in the United States, National Hospital Discharge Survey, 1988-95. Cancer Causes Control 2000;11:497-512.

(Accepted 17 April 2002) 